PROCEEDINGS OF THE

AMERICAN MATHEMATICAL SOCIETY

Volume 132, Number 10, Pages 3069-3074

S 0002-9939(04)07443-X

Article electronically published on June 2, 2004

\title{
COHOMOLOGY OF SYMPLECTIC REDUCTIONS OF GENERIC COADJOINT ORBITS
}

\author{
R. F. GOLDIN AND A.-L. MARE \\ (Communicated by Rebecca Herb)
}

\begin{abstract}
Let $\mathcal{O}_{\lambda}$ be a generic coadjoint orbit of a compact semi-simple Lie group $K$. Weight varieties are the symplectic reductions of $\mathcal{O}_{\lambda}$ by the maximal torus $T$ in $K$. We use a theorem of Tolman and Weitsman to compute the cohomology ring of these varieties. Our formula relies on a Schubert basis of the equivariant cohomology of $\mathcal{O}_{\lambda}$, and it makes explicit the dependence on $\lambda$ and a parameter in $\operatorname{Lie}(T)^{*}=: \mathfrak{t}^{*}$.
\end{abstract}

\section{INTRODUCTION}

Let $K$ be a compact semisimple Lie group, $T \subset K$ a maximal torus and $\mathfrak{t} \subset \mathfrak{k}$ their Lie algebras. Pick a fundamental chamber in $\mathfrak{t}^{*}$ and a point $\lambda$ in the interior. Let $\mathcal{O}_{\lambda}$ be the orbit of $\lambda$ under the coadjoint representation of $K$ on $\mathfrak{k}^{*}$. $\mathcal{O}_{\lambda}$ is diffeomorphic to the flag variety $K / T$, and it has a naturally occurring symplectic form $\omega$ known as the Kirillov-Kostant-Souriau form. The action of $T$ on $\mathcal{O}_{\lambda}$ is Hamiltonian, which means that there is an invariant map

$$
\Phi: \mathcal{O}_{\lambda} \rightarrow \mathfrak{t}^{*}
$$

satisfying $\omega\left(X_{\eta}, \cdot\right)=d \Phi^{\eta}$, where $\eta \in \mathfrak{t}, X_{\eta}$ is the vector field on $\mathcal{O}_{\lambda}$ generated by $\eta$, and $\Phi^{\eta}(m)=\Phi(m)(\eta)$ is defined by the natural pairing between $\mathfrak{t}$ and $\mathfrak{t}^{*}$. We call $\Phi$ a moment map for this action.

The image of $\Phi$ is the convex hull of $W \cdot \lambda$, the Weyl group orbit of $\lambda$. Let $\mu \in \Phi\left(\mathcal{O}_{\lambda}\right)$ be a regular value of $\Phi$. We define the symplectic reduction at $\mu$ by

$$
\Phi^{-1}(\mu) / T=\mathcal{O}_{\lambda} / / T(\mu)
$$

The goal of this note is to give a presentation of the cohomology ring of $\mathcal{O}_{\lambda} / / T(\mu)$ in terms of the root system of $K$. We present $H^{*}\left(\mathcal{O}_{\lambda} / / T(\mu)\right)$ as a quotient of the $T$-equivariant cohomology ring $H_{T}^{*}\left(\mathcal{O}_{\lambda}\right)$ by a certain ideal. We rely on the following fundamental result.

\footnotetext{
Received by the editors November 8, 2002.

2000 Mathematics Subject Classification. Primary 53D20, 14M15.

Key words and phrases. Coadjoint orbits, symplectic reduction, Schubert classes.

The first author was supported by NSF-DMS grant number 0305128 .

${ }^{1}$ Only cohomology with coefficients in the field $\mathbb{Q}$ of rational numbers will be considered throughout this paper.
} 
Theorem 1.1 (Kirwan). Let $M$ be a compact symplectic manifold with a Hamiltonian $T$ action, where $T$ is a compact torus. If $\mu \in \mathfrak{t}^{*}$ is a regular value of $\Phi$, then the restriction map in equivariant cohomology,

$$
\kappa: H_{T}^{*}(M) \rightarrow H_{T}^{*}\left(\Phi^{-1}(\mu)\right)
$$

is surjective.

Since the $T$ action is locally free on level sets of the moment map at regular values, $H_{T}^{*}\left(\Phi^{-1}(\mu)\right)=H^{*}(M / / T(\mu))$. The resulting map $\kappa: H_{T}^{*}(M) \rightarrow$ $H^{*}(M / / T(\mu))$ is called the Kirwan map. Kirwan's result is of particular importance because the equivariant cohomology can be described in terms of the equivariant cohomology of the fixed point sets of the $T$ action. In the case of isolated fixed points, this is just a sum of polynomial rings.

Theorem 1.2 (Kirwan). Let $M$ be a compact Hamiltonian T-space. Let $M^{T}$ denote the fixed point set of the $T$ action. The restriction map

$$
i^{*}: H_{T}^{*}(M) \rightarrow H_{T}^{*}\left(M^{T}\right)
$$

is injective. In the case that $M^{T}$ is a finite set of points,

$$
H_{T}^{*}\left(M^{T}\right)=\bigoplus_{p \in M^{T}} \mathbb{Q}\left[x_{1}, \ldots, x_{n}\right]
$$

where $n=\operatorname{dim} T$.

A presentation of the cohomology ring of the reduced space $M / / T(\mu)$ can be obtained by using the following description of the kernel of the Kirwan map, which is due to Tolman and Weitsman [TW]. If $\alpha$ is in $H_{T}^{*}(M)$ we denote

$$
\operatorname{supp}(\alpha)=\left\{p \in M^{T}:\left.\alpha\right|_{p} \neq 0\right\} .
$$

Fix an arbitrary inner product $\langle$,$\rangle on \mathfrak{t}^{*}$.

Theorem 1.3 (Tolman-Weitsman). The kernel of the Kirwan map $\kappa$ is the ideal of $H_{T}^{*}(M)$ generated by all $\alpha \in H_{T}^{*}(M)$ with the property that there exists $\xi \in \mathfrak{t}^{*}$ such that

$$
\Phi(\operatorname{supp}(\alpha)) \subset\left\{x \in \mathfrak{t}^{*} \mid\langle\xi, x\rangle \leq\langle\xi, \mu\rangle\right\} .
$$

In other words, $\operatorname{ker} \kappa$ consists of sums of equivariant cohomology classes $\alpha$ with the property that all points of supp $(\alpha)$ are mapped by $\Phi$ to the same side of an affine hyperplane in $\mathfrak{t}^{*}$ that passes through $\mu$.

The $T$-equivariant cohomology ring of the coadjoint orbit $\mathcal{O}_{\lambda}=K / T$ is well understood. Kostant and Kumar constructed in [KK] a basis $\left\{x_{w}\right\}_{w \in W}$ of $H_{T}^{*}(K / T)$ as a $H_{T}^{*}(p t)$-module, which we refer to as the Schubert basis. Let $B$ be a Borel subgroup in $G:=K^{\mathbb{C}}$, and let $B_{-}$be the opposite Borel subgroup. For any $v \in W$, let $X_{v}=\overline{B_{-} \tilde{v} B} / B$, where $\tilde{v}$ is any choice of lift of $v \in W$ in the normalizer of the torus. These opposite Schubert varieties are $T$-invariant subvarieties of $G / B \cong$ $K / T$. The basis $\left\{x_{w}\right\}$ is uniquely defined by the property that

$$
\int_{X_{v}} x_{w}=\delta_{v w} .
$$

Theorem 1.2 suggests the importance of knowing how to restrict the classes $x_{w}$ to fixed points $W \cdot \lambda$. This formula was worked out for general $K$ by S. Billey [Bi]. In 
particular, it is easy to show that $\left.x_{w}\right|_{v}=0$ if $v \not \leq w$ in the Bruhat order 2 In other words,

$$
\operatorname{supp}\left(x_{w}\right)=\{v \lambda: v \leq w\} .
$$

To each $\tau \in W$ we can associate the new basis

$$
\left\{x_{w}^{\tau}=\tau \cdot x_{\tau^{-1} w}\right\}_{w \in W},
$$

whose elements have the property

$$
\operatorname{supp}\left(x_{w}^{\tau}\right)=\left\{v \lambda: \tau^{-1} v \leq \tau^{-1} w\right\} .
$$

Let $\lambda_{1}, \ldots, \lambda_{l} \in \mathfrak{t}^{*}$ denote the fundamental weights associated to the chosen fundamental chamber of $\mathfrak{t}^{*}$. Let $\langle$,$\rangle be the restriction to \mathfrak{t}^{*}$ of a $K$-invariant product on $\mathfrak{k}^{*}$. Our main result is:

Theorem 1.4. The cohomology ring $H^{*}\left(\mathcal{O}_{\lambda} / / T(\mu)\right)$ is isomorphic to the quotient of $H_{T}^{*}(K / T)$ by the ideal generated by

$$
\left\{x_{v}^{\tau}: \text { there exists } j \text { such that }\left\langle\lambda_{j}, \tau^{-1} v \lambda\right\rangle \leq\left\langle\lambda_{j}, \tau^{-1} \mu\right\rangle\right\} .
$$

Remark 1. One can take the description of $H_{T}^{*}(K / T)$ (see, for instance, $[\mathrm{Br}]$ ) and deduce a precise presentation of the cohomology ring $H^{*}\left(\mathcal{O}_{\lambda} / / T(\mu)\right)$ in terms of generators and relations.

Remark 2. For $K=S U(n)$ this result was proven by the first author in [Go1].

\section{Primary DESCRIPTION OF ker $\kappa$}

For any $\xi \in \mathfrak{t}^{*}$ we denote by $f_{\xi}$ the corresponding height function on $\mathcal{O}_{\lambda}$,

$$
f_{\xi}(x)=\langle\xi, x\rangle .
$$

Under the pairing between $\mathfrak{t}^{*}$ and $\mathfrak{t}$, the function $f_{\xi}$ is a component of the moment map. In fact, it is well known that $f_{\xi}$ is Morse-Bott for all $\xi \in \mathfrak{t}^{*}$. Denote by $C \subset \mathfrak{t}^{*}$ the fundamental (positive) Weyl chamber, which can be described by

$$
C=\left\{r_{1} \lambda_{1}+\cdots+r_{l} \lambda_{l}: \text { all } r_{j}>0\right\},
$$

and let $\bar{C}$ be its closure.

Lemma 2.1. Let $\tau$ be in $W$ and $\xi$ in $\tau \bar{C}$. If $\tau^{-1} v<\tau^{-1} w$ in the Bruhat order, then $f_{\xi}(v \lambda) \leq f_{\xi}(w \lambda)$.

Proof. The result follows immediately from the fact that if $\xi \in C$, then the unstable manifold of $f_{\xi}$ through $v \lambda$ with respect to the Kähler metric on

$$
\mathcal{O}_{\lambda}=K / T=G / B
$$

is just the Bruhat cell $B \cdot v B / B$ (see, for instance, [Ko]).

\footnotetext{
${ }^{2}$ The class $x_{w}$ differs from the $\xi^{w}$ constructed in [KK] by the relationship $x_{w}:=w_{0} \cdot \xi^{w_{0} w}$, where $w_{0}$ is the longest element of $W$.
} 
The main result of this section is:

Theorem 2.1. Suppose that $\alpha \in H_{T}^{*}\left(\mathcal{O}_{\lambda}\right)$ has the property that

$$
\Phi(\operatorname{supp}(\alpha)) \subset\left\{x \in \mathfrak{t}^{*}:\langle\xi, x\rangle \leq\langle\xi, \mu\rangle\right\} .
$$

Then $\alpha$ can be decomposed as

$$
\alpha=\sum_{w \in W} a_{w}^{\tau} x_{w}^{\tau}
$$

with $a_{w}^{\tau} \in H_{T}^{*}(p t)$, such that if $a_{w}^{\tau} \neq 0$, then

$$
\Phi\left(\operatorname{supp}\left(x_{w}^{\tau}\right)\right) \subset\left\{x \in \mathfrak{t}^{*}:\langle\xi, x\rangle \leq\langle\xi, \mu\rangle\right\} .
$$

Proof. Take $\tau \in W$ such that $\xi \in \tau \bar{C}$. Suppose that the decomposition of $\alpha$ with respect to the basis $\left\{x_{w}^{\tau}\right\}_{w \in W}$ is of the form

$$
\alpha=\sum_{w \in W} a_{w}^{\tau} x_{w}^{\tau}+a_{v_{1}}^{\tau} x_{v_{1}}^{\tau}+\cdots+a_{v_{r}}^{\tau} x_{v_{r}}^{\tau},
$$

where the first sum contains only $w$ with

$$
\langle\xi, w \lambda\rangle \leq\langle\xi, \mu\rangle
$$

whereas

$$
\left\langle\xi, v_{j} \lambda\right\rangle>\langle\xi, \mu\rangle, \quad a_{v_{j}}^{\tau} \in S\left(\mathfrak{t}^{*}\right), a_{v_{j}}^{\tau} \neq 0
$$

for any $1 \leq j \leq l$. We may assume that $v_{1}$ has the property that there exists no $j>1$ with $\tau^{-1} v_{1}<\tau^{-1} v_{j}$. Now let us evaluate both sides of (1) at $v_{1} \lambda$. Since

$$
\langle\xi, w \lambda\rangle \leq\langle\xi, \mu\rangle<\left\langle\xi, v_{1} \lambda\right\rangle
$$

by Lemma 2.1 we must have

$$
\left.x_{w}^{\tau}\right|_{v_{1} \lambda}=0
$$

for any $w$ corresponding to a term in the first sum in (1). It follows that

$$
\left.\alpha\right|_{v_{1} \lambda}=\left.a_{v_{1}}^{\tau} x_{v_{1}}^{\tau}\right|_{v_{1} \lambda} \neq 0
$$

so $v_{1} \lambda$ is in $\operatorname{supp}(\alpha)$ even though $\left.\left\langle\xi, v_{1} \lambda\right\rangle\right\rangle\langle\xi, \mu\rangle$. This is a contradiction.

\section{Proof of the MAIN RESUlt}

We now prove Theorem 1.4. Let $v$ and $\tau$ in $W$ be such that

$$
\left\langle\lambda_{j}, \tau^{-1} v \lambda\right\rangle \leq\left\langle\lambda_{j}, \tau^{-1} \mu\right\rangle,
$$

for some $1 \leq j \leq l$. We show that $x_{v}^{\tau}$ is in the kernel of the Kirwan map

$$
\kappa: H_{T}^{*}\left(\mathcal{O}_{\lambda}\right) \rightarrow H^{*}\left(\mathcal{O}_{\lambda} / / T(\mu)\right)
$$

Let $\xi=\tau \lambda_{j}$ be in $\tau \bar{C}$. Note that if $w \in \operatorname{supp}\left(x_{v}^{\tau}\right)$, then $\tau^{-1} w \leq \tau^{-1} v$ implies by Lemma 2.1 that

$$
\langle\xi, w \lambda\rangle \leq\langle\xi, v \lambda\rangle \leq\langle\xi, \mu\rangle
$$

Thus $x_{v}^{\tau} \in \operatorname{ker} \kappa$.

Now let us consider $\alpha \in H_{T}^{*}(K / T)$ with the property that there exists $\xi \in \mathfrak{t}^{*}$ with

$$
\operatorname{supp}(\alpha) \subset\left\{x \in \mathfrak{t}^{*} \mid\langle\xi, x\rangle \leq\langle\xi, \mu\rangle\right\}
$$


Take $\tau \in W$ such that $\xi \in \tau \bar{C}$. By Theorem 2.2, we can decompose $\alpha$ as

$$
\alpha=\sum_{w \in W} a_{w}^{\tau} x_{w}^{\tau}
$$

where $a_{w}^{\tau}$ can be nonzero only if

$$
\operatorname{supp}\left(x_{w}^{\tau}\right) \subset\left\{x \in \mathfrak{t}^{*} \mid\langle\xi, x\rangle \leq\langle\xi, \mu\rangle\right\} .
$$

In particular, if $a_{w}^{\tau} \neq 0$, then

$$
\langle\xi, w \lambda\rangle \leq\langle\xi, \mu\rangle
$$

Since $\xi$ is in $\tau \bar{C}$, it can be written as

$$
\xi=\tau \sum_{j=1}^{l} r_{j} \lambda_{j}
$$

where all $r_{j}$ are nonnegative. So (4) and (5) imply that there exists $j \in\{1, \ldots, l\}$ such that

$$
\left\langle\tau \lambda_{j}, w \lambda\right\rangle \leq\left\langle\tau \lambda_{j}, \mu\right\rangle
$$

In other words, each nonzero term in the right-hand side of (3) is a multiple of a $x_{w}^{\tau}$ of the type claimed in Theorem 1.4 .

Remark. It follows that, in the particular situation of generic coadjoint orbits, in order to cover the whole Tolman-Weitsman kernel of the Kirwan map it is sufficient to consider affine hyperplanes through $\mu$ that are perpendicular to vectors of the type $\tau \lambda_{j}$, with $\tau \in W$ and $j \in\{1, \ldots, l\}$. But these are just the hyperplanes parallel to the walls of the moment polytope. This result concerning a "sufficient set of hyperplanes" has been proved by the first author in [Go2], for an arbitrary Hamiltonian torus action on a compact manifold.

\section{ACKNowledgement}

The second author would like to thank Lisa Jeffrey for introducing him to the topic of the paper. Both authors would like to thank her for a careful reading of the manuscript and for suggesting several improvements.

\section{REFERENCES}

[Bi] S. Billey, Kostant polynomials and the cohomology of G/B, Duke Math. J. 96 (1999) 205224. MR 2000a:14060

[Br] M. Brion, Equivariant cohomology and equivariant intersection theory, in Representation Theory and Algebraic Geometry, Kluwer Acad. Publ. (1998) 1-37. MR 99m:14005

[Go1] R. F. Goldin, The cohomology ring of weight varieties and polygon spaces, Adv. in Math. 160 (2001) No. 2, 175-204. MR 2002f:53139

[Go2] R. F. Goldin, An effective algorithm for the cohomology ring of symplectic reductions, Geom. and Funct. Anal., Vol. 12 (2002), 567-583. MR 2003m:53148

[Ki] F. C. Kirwan, Cohomology of Quotients in Complex and Algebraic Geometry, Mathematical Notes 31, Princeton University Press, Princeton, N. J. (1984). MR 86i:58050

[Ko] R. R. Kocherlakota, Integral homology of real flag manifolds and loop spaces of symmetric spaces, Adv. in Math. 110 (1995) no. 1, 1-46. MR 96a:57066 
[KK] B. Kostant and S. Kumar, The nil Hecke ring and cohomology of G/P for a Kac-Moody group $G$, Adv. in Math. 62 (1986) no. 3, 187-237. MR 88b:17025b

[TW] S. Tolman and J. Weitsman, The cohomology rings of symplectic quotients Comm. Anal. Geom. 11 (2003), no. 4, 751-773.

Mathematical Sciences, George Mason University, MS 3F2, 4400 University Dr., FairFAX, VIRGINIA 22030

E-mail address: rgoldin@gmu.edu

Department of Mathematics, University of Toronto, Toronto, Ontario, Canada M5S 3G3

E-mail address: amare@math.toronto.edu 